\title{
Speciation Along Environmental Gradients
}

Michael Doebeli (doebeli@zoology.ubc.ca)

Ulf Dieckmann (dieckman@iiasa.ac.at)

\section{Approved by}

Leen Hordijk (hordijk@iiasa.ac.at)

Director, IIASA

December 2002 


\section{IIASA STUDIES IN ADAPTIVE DYNAMICS No. 69}

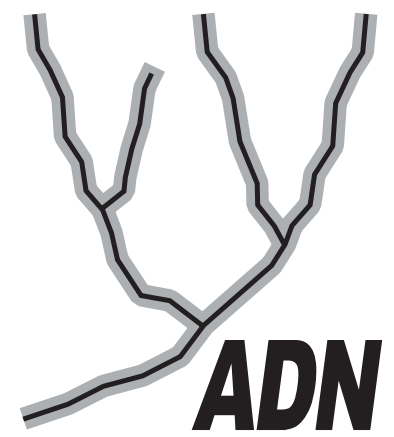

The Adaptive Dynamics Network at IIASA fosters the development of new mathematical and conceptual techniques for understanding the evolution of complex adaptive systems.

Focusing on these long-term implications of adaptive processes in systems of limited growth, the Adaptive Dynamics Network brings together scientists and institutions from around the world with IIASA acting as the central node.

Scientific progress within the network is collected in the IIASA Studies in Adaptive Dynamics series.

No. 1 Metz JAJ, Geritz SAH, Meszéna G, Jacobs FJA, van Heerwaarden JS: Adaptive Dynamics: A Geometrical Study of the Consequences of Nearly Faithful Reproduction. IIASA Working Paper WP-95-099 (1995). van Strien SJ, Verduyn Lunel SM (eds): Stochastic and Spatial Structures of Dynamical Systems, Proceedings of the Royal Dutch Academy of Science (KNAW Verhandelingen), North Holland, Amsterdam, pp. 183-231 (1996).

No. 2 Dieckmann U, Law R: The Dynamical Theory of Coevolution: A Derivation from Stochastic Ecological Processes. IIASA Working Paper WP-96-001 (1996). Journal of Mathematical Biology 34:579-612 (1996).

No. 3 Dieckmann U, Marrow P, Law R: Evolutionary Cycling of Predator-PreyInteractions: Population Dynamics and the Red Queen. IIASA Preprint (1995). Journal of Theoretical Biology 176:91-102 (1995).

No. 4 Marrow P, Dieckmann U, Law R: Evolutionary Dynamics of Predator-Prey Systems: An Ecological Perspective. IIASA Working Paper WP-96-002 (1996). Journal of Mathematical Biology 34:556-578 (1996).

No. 5 Law R, Marrow P, Dieckmann U: On Evolution under Asymmetric Competition. IIASA Working Paper WP-96-003 (1996). Evolutionary Ecology 11:485-501 (1997).

No. 6 Metz JAJ, Mylius SD, Diekmann O: When Does Evolution Optimize? On the Relation Between Types of Density Dependence and Evolutionarily Stable Life History Parameters. IIASA Working Paper WP-96-004 (1996).

No. 7 Ferrière R, Gatto M: Lyapunov Exponents and the Mathematics of Invasion in Oscillatory or Chaotic Populations. Theoretical Population Biology 48:126-171 (1995).

No. 8 Ferrière R, Fox GA: Chaos and Evolution. IIASA Preprint (1996). Trends in Ecology and Evolution 10:480485 (1995).

No. 9 Ferrière R, Michod RE: The Evolution of Cooperation in Spatially Heterogeneous Populations. IIASA Working Paper WP-96-029 (1996). The American Naturalist 147:692717 (1996).

No. 10 van Dooren TJM, Metz JAJ: Delayed Maturation in Temporally Structured Populations with Non-Equilibrium Dynamics. IIASA Working Paper WP-96-070 (1996). Journal of Evolutionary Biology 11:41-62 (1998).
No. 11 Geritz SAH, Metz JAJ, Kisdi É, Meszéna G: The Dynamics of Adaptation and Evolutionary Branching. IIASA Working Paper WP-96-077 (1996). Physical Review Letters 78:2024-2027 (1997).

No. 12 Geritz SAH, Kisdi É, Meszéna G, Metz JAJ: Evolutionary Singular Strategies and the Adaptive Growth and Branching of the Evolutionary Tree. IIASA Working Paper WP-96-114 (1996). Evolutionary Ecology 12:35-57 (1998).

No. 13 Heino M, Metz JAJ, Kaitala V: Evolution of Mixed Maturation Strategies in Semelparous Life-Histories: The Crucial Role of Dimensionality of Feedback Environment. IIASA Working Paper WP-96-126 (1996). Philosophical Transactions of the Royal Society of London Series B 352:1647-1655 (1997).

No. 14 Dieckmann U: Can Adaptive Dynamics Invade? IIASA Working Paper WP-96-152 (1996). Trends in Ecology and Evolution 12:128-131 (1997).

No. 15 Meszéna G, Czibula I, Geritz SAH: Adaptive Dynamics in a 2-Patch Environment: A Simple Model for Allopatric and Parapatric Speciation. IIASA Interim Report IR-97-001 (1997). Journal of Biological Systems 5:265-284 (1997).

No. 16 Heino M, Metz JAJ, Kaitala V: The Enigma of Frequency-Dependent Selection. IIASA Interim Report IR97-061 (1997). Trends in Ecology and Evolution 13:367-370 (1998).

No. 17 Heino M: Management of Evolving Fish Stocks. IIASA Interim Report IR-97-062 (1997). Canadian Journal of Fisheries and Aquatic Sciences 55:1971-1982 (1998).

No. 18 Heino M: Evolution of Mixed Reproductive Strategies in Simple Life-History Models. IIASA Interim Report IR-97063 (1997).

No. 19 Geritz SAH, van der Meijden E, Metz JAJ: Evolutionary Dynamics of Seed Size and Seedling Competitive Ability. IIASA Interim Report IR-97-071 (1997). Theoretical Population Biology 55:324-343 (1999).

No. 20 Galis F, Metz JAJ: Why Are There So Many Cichlid Species? On the Interplay of Speciation and Adaptive Radiation. IIASA Interim Report IR-97-072 (1997). Trends in Ecology and Evolution 13:1-2 (1998). 
No. 21 Boerlijst MC, Nowak MA, Sigmund K: Equal Pay for all Prisoners/ The Logic of Contrition. IIASA Interim Report IR-97-073 (1997). American Mathematical Society Monthly 104:303-307 (1997). Journal of Theoretical Biology 185:281-293 (1997).

No. 22 Law R, Dieckmann U: Symbiosis Without Mutualism and the Merger of Lineages in Evolution. IIASA Interim Report IR-97-074 (1997). Proceedings of the Royal Society of London Series B 265:1245-1253 (1998).

No. 23 Klinkhamer PGL, de Jong TJ, Metz JAJ: Sex and Size in Cosexual Plants. IIASA Interim Report IR-97-078 (1997). Trends in Ecology and Evolution 12:260-265 (1997).

No. 24 Fontana W, Schuster P: Shaping Space: The Possible and the Attainable in RNA Genotype-Phenotype Mapping. IIASA Interim Report IR-98-004 (1998). Journal of Theoretical Biology 194:491-515 (1998).

No. 25 Kisdi É, Geritz SAH: Adaptive Dynamics in Allele Space: Evolution of Genetic Polymorphism by Small Mutations in a Heterogeneous Environment. IIASA Interim Report IR-98-038 (1998). Evolution 53:993-1008 (1999).

No. 26 Fontana W, Schuster P: Continuity in Evolution: On the Nature of Transitions. IIASA Interim Report IR-98-039 (1998). Science 280:1451-1455 (1998).

No. 27 Nowak MA, Sigmund K: Evolution of Indirect Reciprocity by Image Scoring/ The Dynamics of Indirect Reciprocity. IIASA Interim Report IR-98-040 (1998). Nature 393:573-577 (1998). Journal of Theoretical Biology 194:561574 (1998).

No. 28 Kisdi É: Evolutionary Branching Under Asymmetric Competition. IIASA Interim Report IR-98-045 (1998). Journal of Theoretical Biology 197:149-162 (1999).

No. 29 Berger U: Best Response Adaptation for Role Games. IIASA Interim Report IR-98-086 (1998).

No. 30 van Dooren TJM: The Evolutionary Ecology of Dominance-Recessivity. IIASA Interim Report IR-98-096 (1998). Journal of Theoretical Biology 198:519-532 (1999).

No. 31 Dieckmann U, O'Hara B, Weisser W: The Evolutionary Ecology of Dispersal. IIASA Interim Report IR-98-108 (1998). Trends in Ecology and Evolution 14:88-90 (1999).

No. 32 Sigmund K: Complex Adaptive Systems and the Evolution of Reciprocation. IIASA Interim Report IR-98-100 (1998). Ecosystems 1:444-448 (1998).

No. 33 Posch M, Pichler A, Sigmund K: The Efficiency of Adapting Aspiration Levels. IIASA Interim Report IR-98103 (1998). Proceedings of the Royal Society London Series B 266:1427-1435 (1999).

No. 34 Mathias A, Kisdi É: Evolutionary Branching and Coexistence of Germination Strategies. IIASA Interim Report IR-99-014 (1999).

No. 35 Dieckmann U, Doebeli M: On the Origin of Species by Sympatric Speciation. IIASA Interim Report IR-99-013 (1999). Nature 400:354-357 (1999).

No. 36 Metz JAJ, Gyllenberg M: How Should We Define Fitness in Structured Metapopulation Models? Including an Application to the Calculation of Evolutionarily Stable Dispersal Strategies. IIASA Interim Report IR-99-019 (1999). Proceedings of the Royal Society of London Series B 268:499508 (2001)
No. 37 Gyllenberg M, Metz JAJ: On Fitness in Structured Metapopulations. IIASA Interim Report IR-99-037 (1999). Journal of Mathematical Biology 43:545-560 (2001).

No. 38 Meszéna G, Metz JAJ: Species Diversity and Population Regulation: The Importance of Environmental Feedback Dimensionality. IIASA Interim Report IR-99-045 (1999).

No. 39 Kisdi É, Geritz SAH: Evolutionary Branching and Sympatric Speciation in Diploid Populations. IIASA Interim Report IR-99-048 (1999).

No. 40 Ylikarjula J, Heino M, Dieckmann U: Ecology and Adaptation of Stunted Growth in Fish. IIASA Interim Report IR-99-050 (1999). Evolutionary Ecology 13:433-453 (1999).

No. 41 Nowak MA, Sigmund K: Games on Grids. IIASA Interim Report IR-99-038 (1999). Dieckmann U, Law R, Metz JAJ (eds): The Geometry of Ecological Interactions: Simplifying Spatial Complexity, Cambridge University Press, Cambridge, UK, pp. 135-150 (2000).

No. 42 Ferrière R, Michod RE: Wave Patterns in Spatial Games and the Evolution of Cooperation. IIASA Interim Report IR-99-041 (1999). Dieckmann U, Law R, Metz JAJ (eds): The Geometry of Ecological Interactions: Simplifying Spatial Complexity, Cambridge University Press, Cambridge, UK, pp. 318-332 (2000).

No. 43 Kisdi É, Jacobs FJA, Geritz SAH: Red Queen Evolution by Cycles of Evolutionary Branching and Extinction. IIASA Interim Report IR-00-030 (2000).

No. 44 Meszéna G, Kisdi É, Dieckmann U, Geritz SAH, Metz JAJ: Evolutionary Optimisation Models and Matrix Games in the Unified Perspective of Adaptive Dynamics. IIASA Interim Report IR-00-039 (2000). Selection 2:193-210 (2001).

No. 45 Parvinen K, Dieckmann U, Gyllenberg M, Metz JAJ: Evolution of Dispersal in Metapopulations with Local Density Dependence and Demographic Stochasticity. IIASA Interim Report IR-00-035 (2000).

No. 46 Doebeli M, Dieckmann U: Evolutionary Branching and Sympatric Speciation Caused by Different Types of Ecological Interactions. IIASA Interim Report IR-00-040 (2000). The American Naturalist 156:S77-S101 (2000).

No. 47 Heino M, Hanski I: Evolution of Migration Rate in a Spatially Realistic Metapopulation Model. IIASA Interim Report IR-00-044 (2000). The American Naturalist 157:495511 (2001).

No. 48 Gyllenberg M, Parvinen K, Dieckmann U: Evolutionary Suicide and Evolution of Dispersal in Structured Metapopulations. IIASA Interim Report IR-00-056 (2000). Journal of Mathematical Biology 45:79-105 (2002).

No. 49 van Dooren TJM: The Evolutionary Dynamics of Direct Phenotypic Overdominance: Emergence Possible, Loss Probable. IIASA Interim Report IR-00-048 (2000). Evolution 54: 1899-1914 (2000).

No. 50 Nowak MA, Page KM, Sigmund K: Fairness Versus Reason in the Ultimatum Game. IIASA Interim Report IR00-57 (2000). Science 289:1773-1775 (2000).

No. 51 de Feo O, Ferrière R: Bifurcation Analysis of Population Invasion: On-Off Intermittency and Basin Riddling. IIASA Interim Report IR-00-074 (2000). International Journal of Bifurcation and Chaos 10:443-452 (2000).

No. 52 Heino M, Laaka-Lindberg S: Clonal Dynamics and Evolution of Dormancy in the Leafy Hepatic Lophozia Silvicola. IIASA Interim Report IR-01-018 (2001). Oikos 94:525-532 (2001). 
No. 53 Sigmund K, Hauert C, Nowak MA: Reward and Punishment in Minigames. IIASA Interim Report IR-01-031 (2001). Proceedings of the National Academy of Sciences of the USA 98:10757-10762(2001).

No. 54 Hauert C, De Monte S, Sigmund K, Hofbauer J: Oscillations in Optional Public Good Games. IIASA Interim Report IR-01-036 (2001).

No. 55 Ferrière R, Le Galliard J: Invasion Fitness and Adaptive Dynamics in Spatial Population Models. IIASA Interim Report IR-01-043 (2001). Clobert J, Dhondt A, Danchin E, Nichols J (eds): Dispersal, Oxford University Press, pp. 57-79 (2001).

No. 56 de Mazancourt C, Loreau M, Dieckmann U: Can the Evolution of Plant Defense Lead to Plant-Herbivore Mutualism. IIASA Interim Report IR-01-053 (2001). The American Naturalist 158: 109-123 (2001).

No. 57 Claessen D, Dieckmann U: Ontogenetic Niche Shifts and Evolutionary Branching in Size-Structured Populations. IIASA Interim Report IR-01-056 (2001). Evolutionary Ecology Research 4:189-217 (2002).

No. 58 Brandt H: Correlation Analysis of Fitness Landscapes. IIASA Interim Report IR-01-058 (2001).

No. 59 Dieckmann U: Adaptive Dynamics of Pathogen-Host Interacations. IIASA Interim Report IR-02-007 (2002). Dieckmann U, Metz JAJ, Sabelis MW, Sigmund K (eds): Adaptive Dynamics of Infectious Diseases: In Pursuit of Virulence Management, Cambridge University Press, Cambridge, UK, pp. 39-59 (2002).

No. 60 Nowak MA, Sigmund K: Super- and Coinfection: The Two Extremes. IIASA Interim Report IR-02-008 (2002). Dieckmann U, Metz JAJ, Sabelis MW, Sigmund K (eds): Adaptive Dynamics of Infectious Diseases: In Pursuit of Virulence Management, Cambridge University Press, Cambridge, UK, pp. 124-137 (2002).
No. 61 Sabelis MW, Metz JAJ: Perspectives for Virulence Management: Relating Theory to Experiment. IIASA Interim Report IR-02-009 (2002). Dieckmann U, Metz JAJ, Sabelis MW, Sigmund K (eds): Adaptive Dynamics of Infectious Diseases: In Pursuit of Virulence Management, Cambridge University Press, Cambridge, UK, pp. 379-398 (2002).

No. 62 Cheptou P, Dieckmann U: The Evolution of SelfFertilization in Density-Regulated Populations . IIASA Interim Report IR-02-024 (2002). Proceedings of the Royal Society of London Series B 269:1177-1186 (2002).

No. 63 Bürger R: Additive Genetic Variation Under Intraspecific Competition and Stabilizing Selection: A Two-Locus Study. IIASA Interim Report IR-02-013 (2002). Journal of Theoretical Population Biology 61:197-213 (2002).

No. 64 Hauert C, De Monte S, Hofbauer J, Sigmund K: Volunteering as Red Queen Mechanism for Co-operation in Public Goods Games. IIASA Interim Report IR-02-041 (2002). Science 296:1129-1132 (2002).

No. 65 Dercole F, Ferrière R, Rinaldi S: Ecological Bistability and Evolutionary Reversals under Asymmetrical Competition. IIASA Interim Report IR-02-053 (2002). Evolution 56:1081-1090 (2002).

No. 66 Dercole F, Rinaldi S: Evolution of Cannibalistic Traits: Scenarios Derived from Adaptive Dynamics. IIASA Interim Report IR-02-054 (2002).

No. 67 Bürger R, Gimelfarb A: Fluctuating Environments and the Role of Mutation in Maintaining Quantitative Genetic Variation. IIASA Interim Report IR-02-058 (2002). Genetical Research 80:31-46 (2002).

No. 68 Bürger R: On a Genetic Model of Intraspecific Competition and Stabilizing Selection. IIASA Interim Report IR02-062 (2002).

No. 69 Doebeli M, Dieckmann U: Speciation Along Environmental Gradients. IIASA Interim Report IR-02-079 (2002). Nature 421:259-264 (2003).

Issues of the IIASA Studies in Adaptive Dynamics series can be obtained at www.iiasa.ac.at/Research/ADN/Series.html or by writing to adn@iiasa.ac.at. 


\author{
About the Authors \\ Michael Doebeli \\ Departments of Zoology and Mathematics \\ University of British Columbia \\ Vancouver, Canada V6T 1Z4 \\ Ulf Dieckmann \\ Adaptive Dynamics Network \\ International Institute for Applied Systems Analysis \\ A-2361 Laxenburg, Austria
}

\title{
Acknowledgements
}

We thank H. Metz, D. Tautz, G. Meszéna, D. Schluter, E. Knox, O. Leimar, M. Kirkpatrick and T. Barraclough for discussions and comments. The order of authors is reverse alphabetical. 


\title{
Speciation Along Environmental Gradients
}

\author{
Michael Doebeli \\ Ulf Dieckmann
}

Traditional discussions of speciation are based on geographical patterns of species ranges ${ }^{1,2}$. In allopatric speciation, long-term geographical isolation generates reproductively isolated and spatially segregated descendant species ${ }^{1,3}$. In the absence of geographical barriers, diversification is hindered by gene flow ${ }^{1,3,4}$. Yet a growing body of phylogenetic and experimental data suggests that closely related species often occur in sympatry or have adjacent ranges in regions over which environmental changes are gradual and do not prevent gene flow $^{5-14}$. Theory has identified a variety of evolutionary processes that can result in speciation under sympatric conditions ${ }^{15-25}$, with some recent advances concentrating on the phenomenon of evolutionary branching ${ }^{18,23-25}$. Here we establish a link between geographical patterns and ecological processes of speciation by studying evolutionary branching in spatially structured populations. We show that along an environmental gradient, evolutionary branching can occur much more easily than in non-spatial models. This facilitation is most pronounced for gradients of intermediate slope. Moreover, spatial evolutionary branching readily generates patterns of spatial segregation and abutment between the emerging species. Our results highlight the importance of local processes of adaptive divergence for geographical patterns of speciation, and caution against pitfalls of inferring past speciation processes from present biogeographical patterns.

We extended generic Lotka-Volterra models for frequency-dependent competition to individual-based stochastic models of populations occupying a continuous spatial area. In these models, individuals vary in a quantitative trait $u$, e.g. a morphological, behavioral or physiological character. In addition, each individual is characterized by its spatial location $(x, y)$ in a square spatial area. In this area resources are distributed such that for each spatial location $(x, y)$ there is a phenotype $u_{0}$ with maximal carrying capacity. This phenotype varies linearly with one spatial dimension, $u_{0}(x)=a x+b$, which represents the most gradual environmental structure possible; the other spatial dimension $y$ is ecologically neutral (Fig. 1). Such a resource gradient in one spatial dimension could, for example, represent variation in temperature, humidity, soil nutrients, or prey items along an altitudinal gradient. Accordingly, the carrying capacity $K$ depends on the phenotype as well as on the spatial location, and is assumed to be of Gaussian form: if $N_{\sigma}(x)=\exp \left(-\frac{1}{2} x^{2} / \sigma^{2}\right)$ denotes a Gaussian function with variance $\sigma$, then $K=K_{0} \cdot N_{\sigma_{K}}\left(u-u_{0}(x)\right)$. At each spatial location, carrying capacity decreases with phenotypic distance from its maximum at $u_{0}$; the width of this peak is given by $\sigma_{K}$.

We assumed that the strength of competition between two individuals depends on their phenotypic distance, so that competition is strongest between individuals with similar phenotypes, as e.g. when similarly sized individuals prefer similar types of food. We 


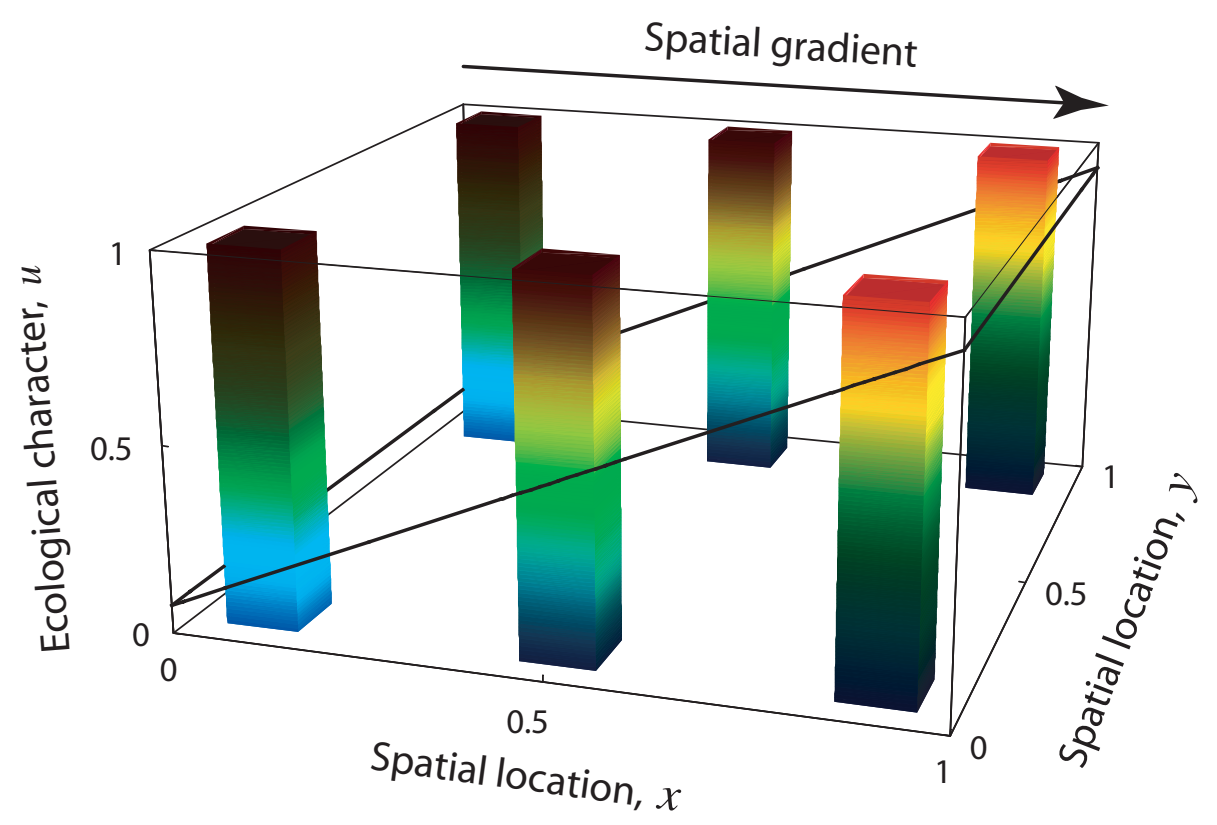

Figure 1: Environmental gradient in carrying capacities. Bright colors correspond to phenotypes that maximize local carrying capacity; these gradually change with spatial location in the $x$-direction, while the $y$-direction is ecologically neutral. At any given location, the carrying capacity decreases with phenotypic distance from the capacitymaximizing phenotype (indicated by diminished brightness).

also assumed that the strength of competition decreases with spatial distance between individuals. Thus, in our individual-based models the effective population size determining the death rate of a given individual due to competition depends both on the number of other individuals in its neighborhood and on their phenotypes. Specifically, in our models the relative strength of competition between two individuals with phenotypic distance $\Delta u$ and spatial distance $d$ is proportional to a product of Gaussian functions: $N_{\sigma_{c}}(\Delta u) \cdot N_{\sigma_{s}}(d)$. The parameters $\sigma_{c}$ and $\sigma_{s}$ determine how fast the intensity of competition declines with phenotypic and spatial distance, respectively. In particular, small values of $\sigma_{s}$ ensure that severe competition is only felt from individuals that are close-by in the spatial arena.

Finally, to describe spatial movement we assumed that individuals move around in the spatial arena with mean movement distances $\sigma_{m}$ and at rates that are independent of location or phenotype. Movement over short distances and localized ecological interactions between individuals allow the population to become spatially structured, whereas frequent movement over long distances tends to result in well-mixed and hence spatially unstructured populations.

Based on these ecological determinants, the evolutionary dynamics of the quantitative trait $u$ were investigated first in asexual populations by allowing for small mutations during birth events. For a variety of reasons (see methods), no reliable analytical or even deterministic theory for the resultant evolutionary dynamics is available at present, so that the direct investigation of individual-based spatially explicit models is necessary. Evolutionary branching of traits determining competitive interactions has already been studied extensively in analytically tractable models without spatial structure ${ }^{18,24,25}$, which can be recovered from our models by setting all spatial coordinates to 0 . In particular, in the nonspatial version of the adaptive dynamics of the quantitative trait $u$, evolutionary branching 
occurs if the strength of competition decreases faster than the resource abundance with phenotypic distance from the capacity-maximizing phenotype, that is, if $\sigma_{c}<\sigma_{K}{ }^{18}$.

Critical aspects of spatial structure are determined by the steepness of the environmental gradient and the movement distance. If the gradient is shallow, the environment becomes essentially spatially homogenous. If movement distances are large, the population becomes well-mixed and hence spatially unstructured. In either of these cases the system's behavior approaches that of the non-spatial model. In particular, evolutionary branching then occurs for the same parameter combinations as in the non-spatial model,that is, for $\sigma_{c}<\sigma_{K}$. When evolutionary branching does occur under such conditions, the two evolving phenotypic clusters are scattered randomly over space.

The system's behavior is dramatically different if the environmental gradient is steep enough and movement distances are short. Evolutionary branching is then accompanied by spatial segregation of the diverging phenotypic clusters (Fig. 2a). Thus, in spatially structured populations evolutionary branching driven by localized and frequency-dependent ecological interactions can generate a sharp phenotypic abutment across a linear environmental gradient. Depending on parameter values, it is possible to observe more than two distinct and spatially segregated lineages (not shown).

A second and perhaps more important effect is that with significant environmental gradients and short movement distances, evolutionary branching occurs for a much wider range of parameters than in the non-spatial models, that is, for values of $\sigma_{c}$ that are much larger than $\sigma_{K}$. The degree to which spatial structure facilitates branching, as well as the abrupt onset of this facilitation as a function of movement distances, are surprising (Fig. 3a). If movement distances exceed a certain threshold value, parameter requirements for branching in the spatial and non-spatial models are almost exactly the same. However, as movement distances are decreased below this threshold, parameter requirements in the spatial model are suddenly and drastically less restrictive than in the non-spatial model. In fact, if the width $\sigma_{s}$ of the spatial interaction kernel is sufficiently small, as is the case in Fig. 3a, evolutionary branching occurs independently of $\sigma_{c}$.

The mechanisms generating these effects can be understood as follows. An environmental gradient initially induces gradual spatial differentiation due to local adaptation along the gradient. Thus, local adaptation results in a correlation between spatial location and phenotype. When, as assumed here, significant competition only occurs between individuals that are spatially sufficiently close, this correlation decreases the strength of competition between phenotypically distant individuals, and hence increases the degree of frequency dependence in the system. This effect tends to disappear if local adaptation is very incomplete due to gene flow along shallow gradients, or if dissimilar phenotypes are spatially close owing to local adaptation along a very steep environmental gradient. Therefore, facilitation of evolutionary branching due to gradient-induced frequency dependence is expected to be highest for intermediate environmental gradients. This is demonstrated in Fig. 3b for a case in which the width $\sigma_{c}$ of the phenotypic interaction is very large, so that frequency dependence is entirely due to localized interactions between spatially differentiated individuals, and no evolutionary branching at all is expected in the non-spatial model. Fig. 3c shows the full characterization of the branching behaviour in asexual populations as a function of the three essential parameters of the model (see methods). It illustrates that for a range of intermediate environmental gradients, evolutionary diversification is greatly facilitated once movement distances or rates fall below a critical level.

We have extended the spatially structured models to sexual populations in which the quantitative character $u$ is determined additively by a number of diploid loci (see 
(a)

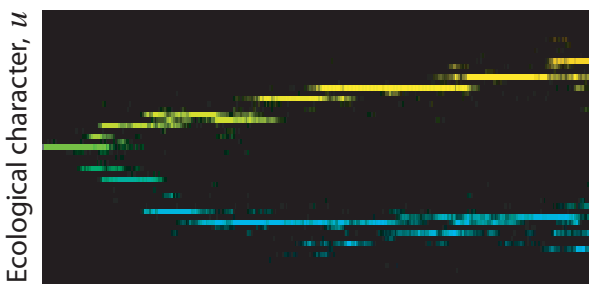

(b)

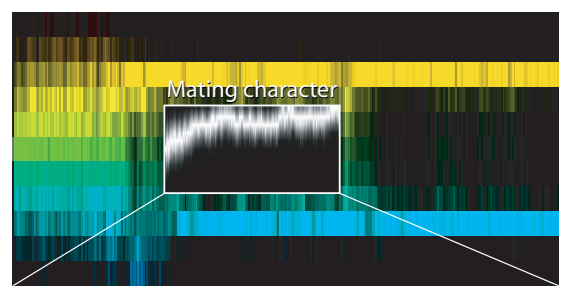

(c)

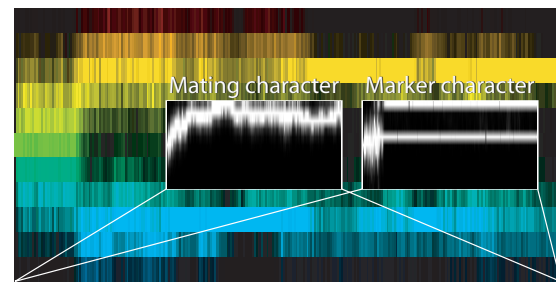

(d)

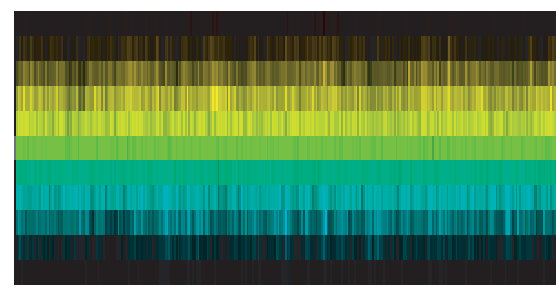

Time, $t$
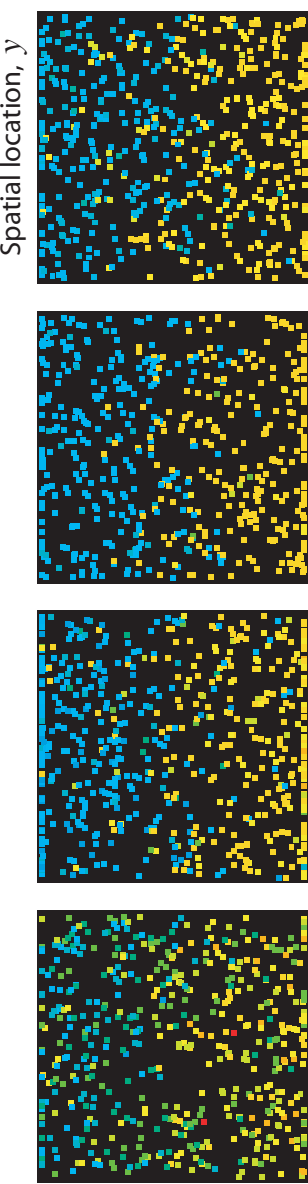

Spatial location, $x$
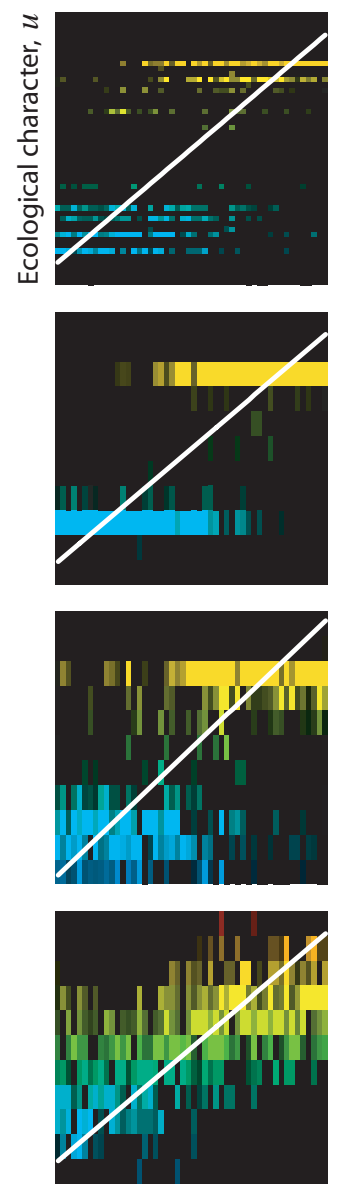

Spatial location, $x$

Figure 2: Evolutionary dynamics of adaptive divergence. In each row the left panels show the distribution of phenotypes as a function of time (same color scheme as in Fig. 1). The middle panels show the distribution of phenotypes across space at the end of the time series, and the right panels show the corresponding frequency distribution of phenotypes as a function of spatial $x$-location. The white lines indicate the environmental gradient (Fig. 1). (a) Evolutionary branching with spatial segregation in an asexual population. See Methods for parameter values. Note that $\sigma_{c}=2.5 \cdot \sigma_{K}$, hence no branching would be expected in the corresponding non-spatial model. (b) Evolutionary branching with spatial segregation in a sexual population with the same parameter values as in (a) and with assortative mating based on ecological similarity. The evolution of the degree of assortativeness is shown as an insert in the left panel (intermediate values of the mating character correspond to random mating, low values to disassortative mating, high values to assortative mating). (c) Evolutionary branching with spatial segregation in a sexual population with assortative mating based on a marker trait. The evolution of assortativeness, as well as the branching in the marker trait, are shown as inserts in the left panel (the two marker branches are in linkage disequilibrium with the two branches of the ecological trait). Parameters values as given in methods, except for $\sigma_{c}=0.5, \sigma_{s}=0.3$ and $\widetilde{\sigma}_{m}=0.38$. (d) Evolution of a phenotypic gradient in a sexual population with random mating. Parameter values are the same as in (b), except that random mating with respect to phenotype was enforced. 

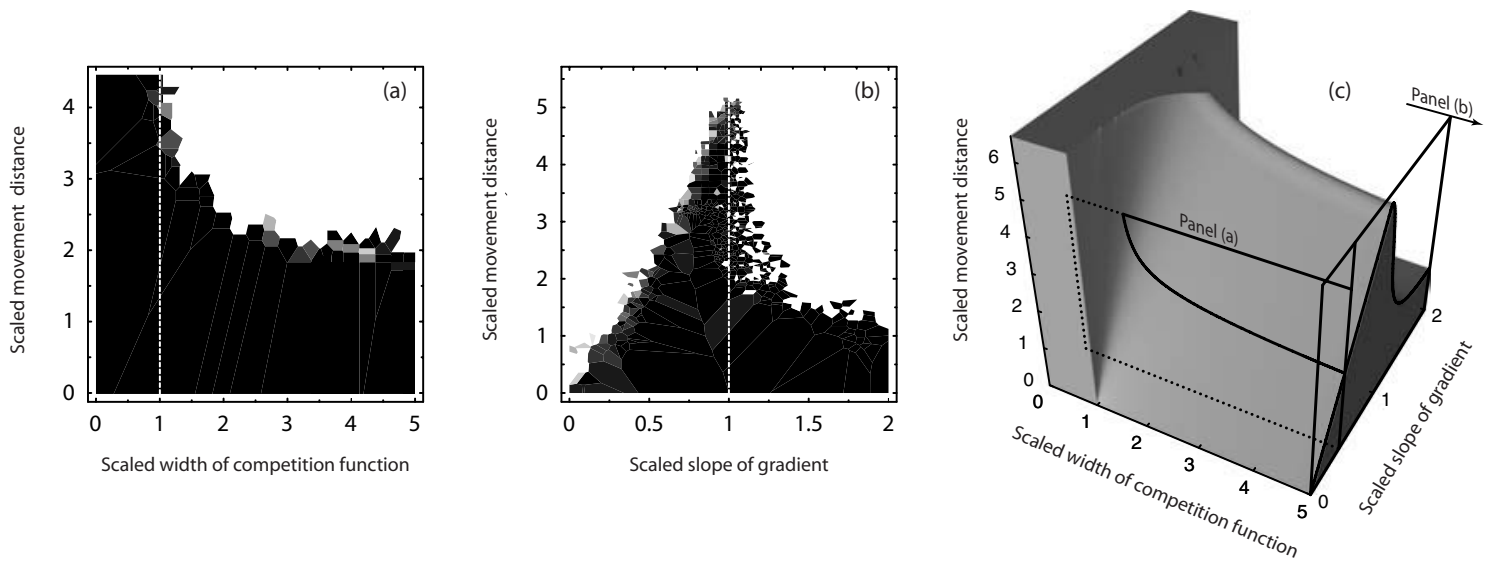

Figure 3: Requirements for spatial evolutionary branching in asexual populations. The model's three dimensionless parameters (see methods) are displayed on all axes: scaled width of competition function $\sigma_{c} / \sigma_{K}$, scaled movement distance $\widetilde{\sigma}_{m} / \sigma_{s}$, and scaled slope of gradient $a \cdot \sigma_{s} / \sigma_{K}$. The first two panels show a subdivision of parameter space into polygons (Voronoi tessellation based on simulation data), shaded according to the recorded time to evolutionary branching: black corresponds to branching within the first 500 generations, white corresponds to no branching after 5000 generations, and shades of gray correspond to branching between generations 500 and 5000 (including multiple branching, which occurs for very small movement distances). (a) Effect of direct frequency dependence. Variation of time until branching with scaled width of competition function and scaled movement distance for asexual populations (at $a \cdot \sigma_{s} / \sigma_{K}=0.425$ ). In non-spatial models ${ }^{18,24}$ only conditions to the left of the dashed line would be expected to induce branching. (b) Effect of gradient-induced frequency dependence. Variation of time until branching with scaled slope of gradient and scaled movement distance for asexual populations (at $\sigma_{c} \gg \sigma_{K}$ ). In non-spatial models no branching would be expected at all. (c) Complete characterization of the asexual model, obtained by extrapolating from Figs. 3a and 3b and from additional numerical simulations. Evolutionary branching occurs for parameters within the shaded block. The positions of slices in panels (a) and (b) are indicated.

methods). The ecological processes remain the same, but instead of reproducing asexually individuals now choose partners within a given spatial neighborhood (see methods). If mating is random with respect to phenotypes, evolutionary branching does not occur anymore, regardless of the choice of parameters. Just as in the non-spatial models, random mating brings about recombination between extremal phenotypes, which prevents the evolution of phenotypic bimodality ${ }^{18}$. By contrast, evolutionary branching is possible in spatially structured sexual populations if one allows for the evolution of assortative mating. Assortative mating can be based on similarity in the primary character determining the ecological interactions, or it can be based on a selectively neutral marker trait ${ }^{18}$. In the latter case, a linkage disequilibrium between the marker trait and the primary trait must evolve for evolutionary branching to occur ${ }^{18}$.

Results for spatially structured sexual populations with assortative mating are in general agreement with those from the asexual models. When evolutionary branching occurs in sexual populations, the emerging phenotypic clusters are essentially reproductively isolated because mating is assortative, and the spatial gradient again separates the emerging species into a pattern of spatial segregation (Fig. 2b,c). Such geographical differentiation 
in the presence of a spatial gradient has been previously observed in a model for competition between two species ${ }^{26}$; this model did not, however, address the question of speciation. It is important to note that, if divergence is prevented by random mating, one expects the evolution of a phenotypic gradient along the environmental gradient ${ }^{2,27,28}$. Such a phenotypic gradient does indeed evolve in our sexual models when individuals mate randomly with regard to their phenotypes (Fig. 2d). Thus, the speciation processes described here are ultimately due to evolutionary branching and to the evolution of assortative mating caused by frequency-dependent interactions under conditions of ecological contact. In sexual populations, speciation through spatial evolutionary branching again occurs for a much wider range of parameters than in the corresponding non-spatial sexual populations, and is most likely with environmental gradients of intermediate slope. The behaviour of the sexual model in which assortative mating is based on similarity in the ecological trait is summarized in Fig. 4. Notice that parameter requirements for spatial evolutionary branching in sexual populations are generally stricter than for asexual populations.

Our results show that intrinsically sympatric processes of adaptive speciation can generate sharp geographic differentiation in the absence of abrupt environmental changes ${ }^{2}, 21$. In traditional models of parapatric speciation due to isolation by distance ${ }^{15,22}$, diversification is driven by divergent local adaptation or genetic drift in spatially distant locations and is hindered by gene flow. In contrast, our models show that ecological contact may in fact be the driving force for parapatric speciation. Gene flow is of course still a hindrance to local divergence, but the mechanisms generating local disruptive selection require ecological contact. Local disruptiveness in turn selects for assortative mating, which reduces and eventually eliminates gene flow between the emerging species. The latter process is akin to reinforcement ${ }^{22}$, but for the fact that selection for prezygotic isolation emerges dynamically from frequency-dependent ecological interactions and is not a consequence of secondary contact. Spatial segregation between closely related species ${ }^{7,13,29}$ is often used to infer allopatric speciation processes. However, our results show that this inference of process from pattern may be misleading, and that instead the origin and distribution pattern of species abutments is consistent with spatial evolutionary branching. This perspective may be important for understanding the origin of species abutments such as those reported for giant senecios along altitudinal gradients ${ }^{8}$, and the origin of hybrid zones such as those between intertidal snails ${ }^{7,13}$.

Our results also show that gradual spatial structure, potentially even more so than complete spatial isolation, facilitates speciation, because local adaptation along an environmental gradient increases the degree of frequency dependence in spatially localized ecological interactions, and hence the likelihood that these interactions generate disruptive selection. Interestingly, this facilitation is most pronounced for environmental gradients of intermediate slope, a result that is fundamentally different from those expected in classical parapatric speciation models. Other things being equal, we can thus hypothesize that speciation rates are highest in populations exposed to environmental gradients with slopes around $\sigma_{K} / \sigma_{s}$ (Figs. 3b,c,4b,c), a measure that varies widely between taxa. This conjecture appears to be testable empirically, both by comparative analyses and by studying the effects of environmental gradients on the experimental evolution of diversity in microorganisms ${ }^{10}$. In sum, the theory presented here offers a new perspective on the importance of geographical structure for the evolution of diversity by showing that spatially localized interactions along environmental gradients can facilitate speciation through frequency-dependent selection and result in patterns of geographical segregation between the emerging species. 

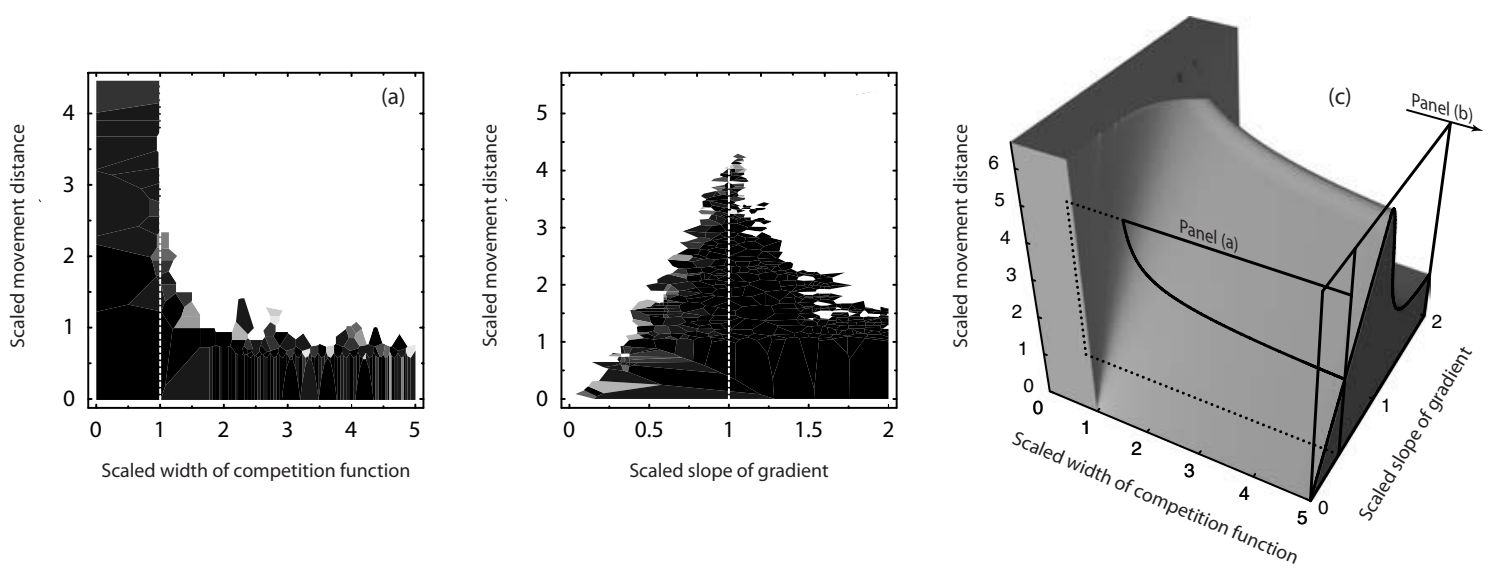

Figure 4: Requirements for spatial evolutionary branching in sexual populations. Same as Fig. 3, but for sexual populations in which assortative mating based on ecological similarity can evolve.

\section{Methods}

Events. Individuals are assigned birth rates $b_{i}$, death rates $d_{i}$, and movement rates $m_{i}, i=1, \ldots, n$, where $n$ is the current population size; these rates are updated as necessary after each event. Time is continuous and generations are overlapping. Based on the total rates $B=\sum_{i=1}^{n} b_{i}, D=\sum_{i=1}^{n} d_{i}, M=\sum_{i=1}^{n} m_{i}$, and $E=B+D+M$, the time lapse until the next event is drawn from an exponential probability distribution with mean $1 / E$; the type of event is chosen according to the probabilities $B / E, D / E$, and $M / E$. The affected individual $i$ is then chosen with probability $b_{i} / B, d_{i} / D$, or $m_{i} / M$, and the chosen individual either gives birth to one offspring, dies, or moves, depending on the event type occurring.

Phenotypes. In the asexual models, ecological phenotypes $0 \leq u \leq 1$ vary continuously. In the sexual models, ecological, mating and marker phenotypes are each determined by $l$ diallelic diploid loci with additive effects and free recombination. Ecological and marker phenotypes vary between 0 and 1 . The mating character varies between -1 (disassortative mating) and +1 (assortative mating); 0 encodes random mating ${ }^{18,24}$.

Gradient. Individuals have a spatial location $(x, y)$, with $0 \leq x, y \leq L$. The carrying capacity for the ecological phenotype $u$ at spatial location $(x, y)$ is $K(u, x, y)=K_{0}$. $N_{\sigma_{K}}\left(u-u_{0}(x)\right)$, where $u_{0}(x)=a\left(x-\frac{L}{2}\right)+\frac{L}{2}$ is the phenotype maximizing $K$ at location $x$, and $0 \leq a \leq 1$ is the slope of the environmental gradient (Fig. 1$) ; u_{0}$ thus varies over space in the range $[(1-a) L / 2,(1+a) L / 2]$.

Death. The effective density experienced by an individual $i$ with phenotype $u$ at location $(x, y)$ is a weighted sum, $n_{\mathrm{eff}}(u, x, y)=\frac{1}{2 \pi \sigma_{s}^{2}} \sum N_{\sigma_{c}}(\Delta u) \cdot N_{\sigma_{s}}(d)$, extending over all pairs $(\Delta u, d)$ of phenotypic and spatial distances between the focal and other individuals. The resultant logistic death rate is $d_{i}=n_{\text {eff }}(u, x, y) / K(u, x, y)$.

Birth. In asexual populations, individuals reproduce at a fixed rate $b_{i}=b$. Offspring express the parental phenotype unless a mutation occurs at probability $\mu_{a}$, in which case their phenotype $u^{\prime}$ is chosen according to $N_{\sigma_{a}}\left(u^{\prime}-u\right)$. For sexual populations, refs. ${ }^{18,24}$ describe how an individual $i$ slated for reproduction chooses a partner $j$ according to phenotype-based mating probabilities $p_{i j}$ depending on its mating character and the partner's phenotypic distance in either ecological or marker character. While this does not yet imply a cost to choosiness (the $p_{i j}$ are normalized), there already is a cost to rarity 
when mating is assortative (individuals with uncommon phenotypes will rarely be chosen as mating partners). In our spatial models, the location-based component $q_{i j}$ of mating probabilities decreases according to $\frac{1}{2 \pi \sigma_{p}^{2}} N_{\sigma_{p}}(d)$ with the spatial distance $d$ between potential partners. This induces a cost to preferring locally rare phenotypes: $b_{i}=b /\left(1+c / n_{p}\right)$, where $n_{p}=\sum_{j=1, j \neq i}^{n} p_{i j} q_{i j}$ is the number of suitable mating partners locally available to individual $i$, and $c$ determines the cost's strength. Notice that assortativeness often evolves despite this cost. (For sexual populations, only females are modeled; males are assumed to have the same density and frequency distributions as females. Given the probabilistic recipe for finding mates, this simplification is uncritical and seems justified in view of otherwise even more computationally demanding sex-structured simulations.) After recombination, the offspring genotype is subjected to allelic mutations according to a reversal probability $\mu_{r}$. Offspring undergo an initial movement event from the location of their parent.

Movement. Individuals move at a fixed rate $m_{i}=m$, undergoing displacements $d$ in the $x$ - and $y$-directions drawn independently according to $N_{\sigma_{m}}(d)$, resulting in an average movement distance $\sigma_{m}$. Boundaries are reflective in the $x$-direction and periodic in the ecologically neutral $y$-direction. For aiding interpretation it is convenient to consider the expected movement distance during the average lifespan of an individual at demographic equilibrium, $\widetilde{\sigma}_{m}=\sqrt{m / b} \cdot \sigma_{m}$ (where $b$ is the birth rate).

Parameters. Unless otherwise stated: $l=10, L=1, K_{0}=500, \sigma_{K}=0.3, a=0.95$, $\sigma_{c}=0.9, \sigma_{s}=0.19, b=1, \mu_{a}=0.005, \sigma_{a}=0.05, \sigma_{p}=0.2, c=10, \mu_{r}=0.001, m=5$, $\widetilde{\sigma}_{m}=0.27$. In the salient limit of large $L, K_{0}$ and small $\mu_{a} \sigma_{a}^{2}$ the asexual model has no more than three essential dimensionless parameters: $\sigma_{c} / \sigma_{K}, \widetilde{\sigma}_{m} / \sigma_{s}$, and $a \cdot \sigma_{s} / \sigma_{K}$ (obtained from choosing units for time, space, and phenotype as $1 / b, \sigma_{s}$, and $\left.\sigma_{K}\right)$. This important simplification allows for a complete characterization of the asexual model as shown in Figs. 3abc. To illustrate the biological meaning of the three dimensionless parameters, we note that if the first, $\sigma_{c} / \sigma_{K}$, is equal to 1 , then the phenotypic distance reducing the strength of competition by a given amount is the same as the phenotypic distance from the capacity-maximizing phenotype reducing the carrying capacity by the same amount; if the second, $\widetilde{\sigma}_{m} / \sigma_{s}$, is equal to 1 , then the expected movement distance during an average lifespan equals $\sigma_{s}$, the width of the spatial interaction kernel; and if the third, $a \cdot \sigma_{s} / \sigma_{K}$, is equal to 1 , then movement of a capacity-maximizing phenotype by $\sigma_{s}$ in the $x$-direction reduces its carrying capacity by $1 / e$.

Approximations. We also investigated approximations of the asexual individualbased model. Although being more tractable by deterministically describing the dynamics of a population density $n(x, u)$, these approximations are problematic. First, such continuum approximations are based on the limit of infinite local population sizes (local both in $x$ and $u$ ), which is even more difficult to justify biologically than the limit of infinite global population size, widely used in population ecology. Second, a conveniently simple reaction-diffusion approximation of this system, derived for small $\sigma_{m}$ and $\sigma_{s}$, is dynamically unstable. Third, these approximations ignore the implications of reproductive (and other) pair correlations and local density fluctuations, both of which have been shown to critically affect ecological and evolutionary dynamics ${ }^{30}$. Fourth, the deterministic approximation blurs the sharp bifurcation boundary in Fig. 3a and is also inaccurate in predicting the boundary's location. Fifth, extending the deterministic approximation to multilocus genetics is not feasible without incurring further unjustified assumptions. 


\section{References}

1. Mayr, E. Animal Species and Evolution (Harvard University Press, Cambridge, MA, 1963).

2. Endler, J. A. Geographic variation, speciation, and clines (Princeton University Press, Princeton, NJ, 1977).

3. Coyne, J. A. Genetics and speciation. Nature 355, 511-515 (1992).

4. Felsenstein, J. Scepticism towards Santa Rosalia, or why are there so few kinds of animals. Evolution 35, 124-138 (1981).

5. Schliewen, U. K. Tautz, D. \& Pääbo, S. Sympatric speciation suggested by monophyly of crater lake cichlids. Nature 368, 629-632 (1994).

6. Schluter, D. Experimental evidence that competition promotes divergence in adaptive radiation. Science 266, 798-801 (1994).

7. Johannesson, K., Rolán-Alvarez, E. \& Ekendahl, A. Incipient reproductive isolation between two sympatric morphs of the intertidal snail Littorina saxatilis. Evolution 49, 1180-1190 (1995).

8. Knox, E. B. \& Palmer, J. D. Chloroplast DNA variation and the recent radiation of giant senecios (Asteraceae) on the tall mountains of Eastern Africa. Proc. Natl. Acad. Sci. USA 92, 10349-10353 (1995).

9. Feder, J. L. The apple maggot fly, Rhagoletis pomonella: Flies in the face of conventional wisdom about speciation? pp. 130-144 in Endless Forms, D. J. Howard and S. H. Berlocher, Eds. (Oxford University Press, Oxford, UK, 1998).

10. Rainey, P. B. \& Travisano, M. Adaptive radiation in a heterogeneous environment. Nature 394, 69-72 (1998).

11. Gislason, D., Ferguson, M., Skulason, S. \& Snorrason, S. S. Rapid and coupled phenotypic and genetic divergence in Icelandic Arctic char (Salvelinus alpinus). Can. J. Fish. Aquat. Sci. 56, 2229-2234 (1999).

12. Schliewen, U. K., Rassmann, K., Markmann, M., Markert, J. \& Tautz, D. Genetic and ecological divergence of a monophyletic cichlid species pair under fully sympatric conditions in Lake Ejagham, Cameroon. Mol. Ecol. 10, 1471-1488 (2001).

13. Wilding, C. S., Butlin, R. K. \& Grahame, J. Differential gene exchange between parapatric morphs of Littorina saxatilis detected using AFLP markers. J. Evol. Biol. 14, 611-618 (2001).

14. Via, S. Sympatric speciation in animals: the ugly duckling grows up. Trends Ecol Evol. 16, 381-390 (2001)

15. Lande, R. Rapid origin of sexual isolation and character divergence in a cline. Evolution 36, 213-223 (1982).

16. Kawecki, T. J. Sympatric speciation via habitat specialization driven by deleterious mutations. Evolution 51, 1751-1763 (1997). 
17. Kondrashov, A. S. \& Kondrashov, F. A. Interactions among quantitative traits in the course of sympatric speciation. Nature 400, 351-354 (1999).

18. Dieckmann, U. \& Doebeli, M. On the origin of species by sympatric speciation. Nature 400, 354-357 (1999).

19. Van Doorn, G. S., Luttikhuizen, P. C. \& Weissing F. J. Sexual selection at the protein level drives the extraordinary divergence of sex-related genes during sympatric speciation. Proc. Roy. Soc. Lond. B 268, 2155-2161 (2001).

20. Gavrilets, S., and Waxman, D. Sympatric speciation by sexual conflict. Proc. Nat. Acad. Sci. 99, 10533-10538 (2002).

21. Mizera, F., \& Meszéna, G..Spatial niche packing, character displacement and adpative speciation in an environmental gradient. Evol. Ecol. Res. (in press)

22. Turelli, M., Barton, N. H. \& Coyne, J. A. Theory and speciation. Trends Ecol Evol. 16, 330-343 (2001).

23. Geritz, S. A. H., Kisdi, E., Meszéna, G. \& Metz, J. A. J. Evolutionarily singular strategies and the adaptive growth and branching of the evolutionary tree. Evol. Ecol. 12, 35-57 (1998).

24. Doebeli, M. \& Dieckmann, U. Evolutionary branching and sympatric speciation caused by different types of ecological interactions. Am. Nat. 156, S77-S101 (2000).

25. Kisdi, E. Evolutionary branching under asymmetric competition. J. Theor. Biol. 197, 149-162 (1999).

26. Case, T. J. \& Taper, M. L. Interspecific competition, environmental gradients, gene flow, and the coevolution of species' borders. Am. Nat. 155, 583-605 (2000).

27. Slatkin, M. Spatial patterns in the distribution of polygenic characters. J. Theor. Biol. 70, 213-228 (1978).

28. Barton, N. H. Clines in polygenic traits. Genet. Res., Camb. 74, 223-236 (1999).

29. Barraclough, T. G. \& Vogler, A. P. Detecting the geographical pattern of speciation from species-level phylogenies. Am. Nat. 155, 419-434 (2000).

30. Dieckmann, U., Law R. \& Metz J. A. J. The Geometry of Ecological Interactions: Simplifying Spatial Complexity (Cambridge University Press, Cambridge, UK, 2000). 